\title{
A THEOREM ON QUASI-COMPACT MAPPINGS ${ }^{1}$
}

\section{PAUL MCDOUGLE}

1. Introduction. The ascertainment of conditions for topological invariances under various types of mappings, especially where the domain space is metric and the mappings are closed or quasi-compact, has received a considerable amount of recent attention. Necessary and sufficient conditions for the invariance of metrizability under closed mappings have been obtained independently by $\mathrm{K}$. Morita and S. Hanai [1] and by A. H. Stone [2]. A similar type theorem for quasi-compact mappings on locally compact, separable metric spaces is given in the present paper. Previous results in this direction have been obtained by A. V. Martin [3], W. E. Malbon [4] and Stone [2].

2. Definitions. Let $f(X)=Y$ where $X, Y$ are topological spaces and $f$ is a continuous mapping. Then $f$ is a quasi-compact mapping provided that the image of every open inverse set in $X$ is open in $Y$. The mapping $f$ is a $P_{1}$ mapping provided that whenever $y \in Y$ and $U$ is a neighborhood of $f^{-1}(y)$ then $y \in$ int $f(U) ; f$ is a $P_{2}$ mapping provided that for each $y$ in $Y$ there is a compact set $C \subset f^{-1}(y)$ such that whenever $U$ is a neighborhood of $C$ then $y \in$ int $f(U)$. The mapping $f$ is semi-closed provided that whenever $C$ is a compact subset of $X$, then $f(C)$ is closed. The space $X$ is an $M$ space provided that whenever $\left(x_{i}\right) \rightarrow x$ and $\left(x_{i}\right) \rightarrow x^{\prime}$ in $X$, then $x=x^{\prime}$.

3. THEOREM. Let $f$ be a quasi-compact mapping of a locally compact, separable metric space $X$ onto the topological space $Y$; then $Y$ is a locally compact, separable metric space if and only if $f$ is a semi-closed, $P_{2}$ mapping.

It will be convenient to prove several lemmas before establishing this theorem. It may be observed from the definitions that every $P_{2}$ mapping is a $P_{1}$ mapping, and every $P_{1}$ mapping is quasi-compact. Throughout this paper, $f$ will denote a continuous mapping of a topological space $X$ onto a topological space $Y$.

Presented to the Society, October 26, 1957; received by the editors October 4, 1957.

1 This research was supported by the United States Air Force through the Air Force Office of Scientific Research of the Air Research and Development Command, under contract No. AF 49(638)-72 at the University of Virginia.

The author wishes to thank Professor G. T. Whyburn for his advice and encouragement. 
Lemma 1. If $f(X)=Y$, then $f$ is $P_{1}$ if and only if whenever $y \in \bar{A}$ $-A \subset Y$, then $f^{-1}(y) \cdot \mathrm{Cl}\left(f^{-1}(A)\right) \neq 0$.

Proof. Let $f(X)=Y$ be $P_{1}$ and suppose $y \in \bar{A}-A$ where $f^{-1}(y)$ - $\mathrm{Cl}\left(f^{-1}(A)\right)=0$; then for each $x$ in $f^{-1}(y)$ there is a neighborhood $U_{x}$ of $x$ such that $U_{x} \cdot f^{-1}(A)=0$. Let $U=\sum U_{x}$, summed over all $x$ in $f^{-1}(y)$; then $U$ is a neighborhood of $f^{-1}(y)$ and $y$ is not interior to $f(U)$, giving a contradiction.

To prove the sufficiency, suppose $y \in Y, U$ is a neighborhood of $f^{-1}(y)$, and $y$ is not interior to $f(U)$. If $A=Y-f(U)$, then $y \in \bar{A}-A$ and $f^{-1}(y) \cdot \mathrm{Cl}\left(f^{-1}(A)\right)=0$; this gives a contradiction.

Lemma 2. Let $f(X)=Y$ be a $P_{1}$, semi-closed mapping of a metric space $X$ onto $Y$; then $Y$ is an $M$ space.

Proof. $Y$ is $T_{1}$ since $f$ is semi-closed. Let $\left(y_{i}\right) \rightarrow y$ in $Y$; it must be shown that if $y^{\prime} \in Y-y$ then it is false that $\left(y_{i}\right) \rightarrow y^{\prime}$. By application of Lemma 1 and the metrizability of $X$, it follows that there is a sequence of points $\left(x_{i}\right)$ in $f^{-1}\left(\sum y_{i}-y-y^{\prime}\right)$ and an $x$ in $f^{-1}(y)$ such that $\left(x_{i}\right) \rightarrow x$. Then $\left(\sum x_{i}+x\right)$ is a compact set and hence $f\left(\sum x_{i}+x\right)$ is a closed infinite subset of $\left(\sum y_{i}+y-y^{\prime}\right)$; therefore $\left(y_{i}\right) \rightarrow y^{\prime}$ is false.

Lemma 3. Let $f(X)=Y$ be a $P_{2}$, semi-closed mapping of a metric space $X$ onto $Y$; then $Y$ is a Hausdorff space with a locally countable basis.

Proof. Let $y \in Y$ and choose a compact set $C \subset f^{-1}(y)$ such that if $U$ is a neighborhood of $C$ then $y \in \operatorname{int} f(U)$. Let $S_{k}(C)$ denote the $1 / k$ neighborhood of $C$, and let $W_{k}=\operatorname{int} f\left(S_{k}\right), k=1,2, \cdots$. If $W$ is a neighborhood of $y$ and $\rho$ is a metric for $X$, then $\rho\left(C, \operatorname{Fr} f^{-1}(W)\right)$ $>1 / k$ for some positive integer $k$ and hence $W_{k} \subset W$. This proves $\left(W_{k}\right)$ is a basis at $y$; since $Y$ is an $M$ space by Lemma 2 , it follows that $Y$ is also Hausdorff $[4,1.3]$. For if $\left(W_{i}\right),\left(W_{i}^{\prime}\right), i=1,2, \cdots$ were respectively decreasing bases at points $y$ and $y^{\prime}$ in $Y$ not having disjoint neighborhoods, then by choosing $y_{i}$ in $W_{i} \cdot W_{i}^{\prime}$ there results a sequence $\left(y_{i}\right)$ such that $\left(y_{i}\right) \rightarrow y,\left(y_{i}\right) \rightarrow y^{\prime}$ and this gives a contradiction.

The sufficiency of the $P_{2}$ and semi-closed conditions in the theorem now follows by Lemma 3 and a theorem proved by A. H. Stone [2; Theorem 3] to the effect that a Hausdorff space with a locally countable basis is a locally compact, separable metric space if it is the image under a quasi-compact mapping of a locally compact, separable metric space.

In regard to the necessity of the $P_{2}$ and semi-closed conditions in the theorem, it is evident that $f$ is semi-closed. Suppose $f$ is not a $P_{2}$ 
mapping. Then there is a $y \in Y$ such that for each compact set $C \subset f^{-1}(y)$ there is a neighborhood $U$ of $C$ such that $y$ is not interior to $f(U)$. By application of local compactness and the Lindelöf property, there is a representation $f^{-1}(y)=\sum_{k=1}^{\infty} C_{k}$, where $C_{k}$ are compact nonempty sets and $C_{k+1} \supset C_{k}$. Choose $U_{1}$ a conditionally compact neighborhood of $C_{1}$ such that $y$ is not interior to $f\left(U_{1}\right)$, and let $U_{k}$ be inductively defined as a conditionally compact neighborhood of $C_{k}+f^{-1}(y) \cdot \mathrm{Cl}\left(U_{k-1}\right)$ such that $y$ is not interior to $f\left(U_{k}\right)$. Let $V_{k}$ $=\sum_{i=1}^{k} U_{i}, k=1,2, \cdots$; it will now be shown that $y$ is not interior to $f\left(V_{k}\right), k=1,2, \cdots$. Suppose $y \in \operatorname{int} f\left(V_{k}\right)$ for some $k$; then there is a sequence $\left(y_{i}\right) \rightarrow y$ in $Y$ where $y_{i}$ is in $f\left(V_{k}\right)-f\left(U_{k}\right), i=1,2, \cdots$. If $x_{i} \in f^{-1}\left(y_{i}\right) \cdot V_{k}$, the sequence $\left(x_{i}\right)$ has a limit point $x$ in $\mathrm{Cl}\left(V_{k-1}\right)$ and $f(x)=y$ by the continuity of $f$. Hence $x \in f^{-1}(y) \cdot \mathrm{Cl}\left(V_{k-1}\right)$ $=f^{-1}(y) \cdot \mathrm{Cl}\left(U_{k-1}\right) \subset U_{k}$, contradicting the fact that $x$ is a limit point of $\left(x_{i}\right)$ and $x_{i}$ is not in $U_{k}, i=1,2, \cdots$. This proves $y$ is not interior to $f\left(V_{k}\right)$ for $k=1,2, \cdots$.

Since by Lemma 1, Theorem 3 of Stone [2, p. 694], $y$ is interior to $f\left(V_{k}\right)$ for some $k$, there is a contradiction and therefore $f$ is a $P_{2}$ mapping.

The $P_{1}$ and $P_{2}$ properties of mappings are equivalent whenever all point inverses are compact. Included among the $P_{1}$ mappings are all open and all closed mappings [4, pp. 43-45]. For if $f$ is open then $f$ is clearly $P_{1}$; if $f$ is closed and $U$ is a neighborhood of $f^{-1}(y)$ then $f(X-U)$ is closed and therefore $y \in$ int $(Y-f(X-U)) \subset$ int $f(U)$. Hence it is easily seen that all open mappings and all closed mappings with compact point inverses are $P_{2}$ mappings.

The following, a modification of an example due to Malbon [4, Ex. 2.28], shows that $X$ must be assumed locally compact in the theorem. In the plane: for $0<x \leqq 1$ let $A_{x}$ be the line segment $x=x$, $1 \leqq y \leqq 2$ and $p_{x}$ the point $(x, 0)$; let $q$ be the point $(0,1)$. Now let $X=\sum\left(A_{x}+p_{x}\right)+q$ for $0<x \leqq 1$, with the usual topology of the plane, be decomposed as follows. Elements are $p_{x}$ and $A_{x}$ for $x=1 / i$, $i=1,2, \cdots, p_{x}+A_{x}$ for $x$ otherwise, and $q$. Let $Y$ denote the induced decomposition space and $f$ the natural map. Then $X$ is separable metric, though not locally compact at $q$, and $f$ is open, semi-closed, and has compact point inverses. Therefore $Y$ is Hausdorff with a locally countable basis by Lemma 3 . If $P=\sum_{i=1}^{\infty} p_{1 / i}$, then $P$ is a closed subset of $Y$ and $P$ and $q$ do not have disjoint neighborhoods in $Y$. Therefore $Y$ is not regular and hence not separable metric.

\section{BIBLIOGRAPHY}

1. K. Morita and S. Hanai, Closed mappings and metric spaces, Proceedings of the Japanese Academy vol. 32 (1956) pp. 10-14. 
2. A. H. Stone, Metrizability of decomposition spaces, Proc. Amer. Math. Soc. vol. 7 (1956) pp. 690-700.

3. A. V. Martin, Decompositions and quasi-compact mappings, Duke Math. J. vol. 21 (1954) pp. 463-469.

4. W. E. Malbon, Invariants for quasi-compact mappings, University of Virginia Dissertation, 1955.

UNIVERSITY OF VIRGINIA

\section{CONNECTED SETS OF VAN VLECK}

PAUL M. SWINGLE

Both Vitali and Van Vleck have given interesting constructions of Lebesgue nonmeasurable sets in euclidean $E_{1}$. Here we give a generalization for the construction of Van Vleck for $E_{m}, m \geqq 2$; our interest is in the type of connected set that can be so obtained. Elsewhere we will consider the construction of Vitali. Of interest also is the interlacing of these connected sets.

Below $\Omega$ is the first transfinite ordinal whose cardinal is the same as that of the linear continuum: $\alpha, \beta, \gamma$ are ordinals, $>0$ and $<\Omega$. We will say that the Van Vleck basic set for a given point $P_{\alpha}$ $=\left(x_{1 \alpha}, x_{2 \alpha}, \cdots, x_{m \alpha}\right)$ in $\left(x_{1}, x_{2}, \cdots, x_{m}\right)$-coordinate space is the set of all $\left(x_{1 \alpha}^{\prime}, x_{2 \alpha}^{\prime}, \cdots, x_{m \alpha}^{\prime}\right)$ where for each $j(j=1,2, \cdots, m)$, we have as in $[1$, p. 240],

$$
x_{j \alpha}^{\prime}=\frac{x_{j \alpha}}{2^{( \pm p)}} \pm \frac{u}{2^{v}} \quad(u, v, p=0,1,2, \cdots) .
$$

Van Vleck constructs two complementary sets in $E_{1}$, each a reflection about $x=1 / 2$ of the other; it is in part because measure is invariant under reflection that his construction gives Lebesgue nonmeasurable sets.

We will take the Van Vleck $x_{1}$-reflected set for $P_{\alpha}$ as the set of all $\left(-x_{1 \alpha}^{\prime}, x_{2 \alpha}^{\prime}, \cdots, x_{m \alpha}^{\prime}\right)$ with $x_{j \alpha}^{\prime}$ as above, i.e. it is the Van Vleck basic set for $P_{\alpha}$ reflected about $x_{1}=0$; and the $\left(x_{h}, x_{g}, \cdots, x_{k}\right)$-reflected set for $P_{\alpha}$ is the Van Vleck basic set for $P_{\alpha}$ reflected about the coordinate subspace where $x_{h}=0, x_{g}=0, \cdots, x_{k}=0$, i.e. the set of all $\left(x_{1 \alpha}^{\prime \prime}, x_{2 \alpha}^{\prime \prime}, \cdots, x_{m \alpha}^{\prime \prime}\right)$, where $x_{t \alpha}^{\prime \prime}=-x_{t \alpha}^{\prime}$ for $t=h, g, \cdots, k$ and otherwise $x_{t \alpha}^{\prime}=x_{t \alpha}^{\prime \prime}$ : these reflections include one about the origin, referred to as the $(m-m)$-coordinate space below.

Presented to the Society, November 30, 1957; received by the editors October $10,1957$. 